\title{
Color Display for Hyperspectral Imagery
}

\author{
Qian Du, Senior Member, IEEE, Nareenart Raksuntorn, Student Member, IEEE, \\ Shangshu Cai, Student Member IEEE, and Robert J. Moorhead, II, Senior Member, IEEE
}

\begin{abstract}
This paper investigates RGB color composition schemes for hyperspectral imagery display. A three-channel composite inevitably loses a significant amount of information contained in the original high-dimensional data. The objective here is to display the useful information as distinctively as possible for high-class separability. To achieve this objective, it is important to find an effective data processing step prior to color display. A series of supervised and unsupervised data transformation and classification algorithms are reviewed, implemented, and compared for this purpose. The resulting color displays are evaluated in terms of class separability using a statistical detector and perceptual color distance. We demonstrate that the use of the data processing step can significantly improve the quality of color display, whereas data classification generally outperforms data transformation, although the implementation is more complicated. Several instructive suggestions for practitioners are provided.
\end{abstract}

Index Terms-Classification, color display, human visual perception, hyperspectral imaging, transformation, visualization.

\section{INTRODUCTION}

$\mathbf{H}$ YPERSPECTRAL imaging, also known as imaging spectrometry, has become a core technology in remote sensing. The resulting hyperspectral imagery has very high spectral resolution, providing better diagnostic capability for object detection, classification, and discrimination than multispectral imagery. However, it is challenging to display all the useful information contained in such a huge 3-D image cube. A common practice is to use a red-green-blue (RGB) color representation to provide a quick overview of a scene for decision-making support. Obviously, such a three-color channel display results in significant loss of information. Our objective is to display classes distinctively to maximize the class separability.

False-color images, which map three original spectral bands into three RGB channels, have been used to display multispectral images for decades [1]-[4]. For instance, a color infrared (CIR) composite is a typical way to represent vegetation and its vigor from multispectral data. Although CIR provides a simple synoptic view of the scene, the contrast may be poor when the three original channels are highly correlated [3]. When the original data have more than three bands, careful band selection

Manuscript received April 20, 2007; revised November 2, 2007. This research was supported in part by the NASA Science Mission Directorate, Earth System Division, Applied Sciences Program as a Crosscutting Solutions contract to Mississippi State University through the Stennis Space Center.

The authors are with the Department of Electrical and Computer Engineering and the GeoResources Institute in the High Performance Computing Collaboratory $\left(\mathrm{HPC}^{2}\right)$, Mississippi State University, Mississippi State, MS 39762 USA.

Color versions of one or more of the figures in this paper are available online at http://ieeexplore.ieee.org.

Digital Object Identifier 10.1109/TGRS.2008.916203 is required [5]. This technique is referred to as the three-band combination (TBC) method in this paper. It may not be suitable to display a hyperspectral image with hundreds of spectral bands.

One approach for hyperspectral image display is to condense the information into three channels and map the three channels into the RGB color space. A typical method is to perform principal component analysis (PCA) and display the first three principal components (PCs) [6]-[8]. However, PCs may not be ranked in terms of information content, and objects may be present in other PCs with a lower ranking [9]. Thus, a noise-adjusted principal component analysis (NAPCA) may be a better choice, where PCs are ranked in terms of signal-tonoise ratio (SNR) and a $\mathrm{PC}$ with a higher ranking generally contains more object information [10]. When the desired objects are known, a supervised transform called interference and noise-adjusted principal component analysis (INAPCA) can be used [11]. Other transformation-based color display approaches include Jacobson et al.'s work [12], [31], where fixed linear spectral weighting envelopes are used to create natural-looking imagery that displays the same materials in different data sets consistently. The weighting envelopes are designed to take into account how the hardware displays information and the human perceptual system. However, such fixed linear projections will be unable to display some different objects distinctively because there is no adaptation to specific image information.

Another way to display hyperspectral images is to visualize data analysis results using detection, classification, or quantification maps. For a typical pure pixel analysis result, a false-color display can be generated by assigning a color label to each class. Because most pixels are mixed in a remotely sensed hyperspectral image due to low spatial resolution, it is more reasonable to conduct mixed-pixel analysis. In mixedpixel classification, a pixel has a certain percentage of each class. According to our study, images produced using data analysis results are more informative than ones produced using a simple data transformation, because different objects can be more easily differentiated from each other. However, their implementation may be more complicated, particularly under an unsupervised circumstance.

In this paper, we investigate the use of several data transformation and classification approaches for hyperspectral image color display. Specifically, the transformation-based approaches we propose are NAPCA, INAPCA, Fisher's linear discriminant analysis (LDA), and the data classification-based approaches we propose are independent component analysis (ICA), constrained linear discriminant analysis (CLDA), and unsupervised fully constrained linear unmixing (UFCLU). They will be compared with other more frequently used 
approaches, such as PCA, TBC, and the color-matching function (CMF) projection in [12], in terms of class separability in the produced color display. Based on experimental results, instructive suggestions are concluded for practitioners.

The paper is organized as follows. Section II reviews the data transformation and classification techniques. Section III discusses the issues on color display generation, such as color assignment and final color reproduction. Section IV presents experimental results with quantitative evaluation. Finally, Section V draws the conclusions.

\section{Data Processing Techniques}

There exist quite a few data processing techniques for hyperspectral imagery. In this section, we review the rationales befind the seven techniques to be used in this research. They are selected either because they are widely used or because they are closely related to some widely used techniques. In addition to PCA, the CMF technique in [12] and the simplest TBC method are included for comparsion purposes. The conclusions drawn from these techniques are suitable to other similar approaches in hyperspectral data color display.

\section{A. PCA}

Let $\Sigma$ be the data covariance matrix for an L-band hyperspectral image. Let $\mathbf{V}=\left[\mathbf{v}_{1}, \mathbf{v}_{2}, \cdots, \mathbf{v}_{L}\right]$ and $\Lambda=$ $\operatorname{diag}\left\{\lambda_{1}, \lambda_{2}, \cdots, \lambda_{L}\right\}$ be the eigenvector and eigenvalue matrices of $\Sigma$, respectively, which can be related as

$$
\mathbf{V}^{T} \mathbf{\Sigma} \mathbf{V}=\boldsymbol{\Lambda}
$$

Then the PC images can be calculated by

$$
\mathbf{z}_{P C A}=\mathbf{V}^{T}(\mathbf{z}-\mathbf{m})
$$

where $\mathbf{m}$ is the data mean, and $\mathbf{z}$ and $\mathbf{z}_{P C A}$ are pixel vectors before and after the transformation, respectively.

As discussed in [12], there are many problems when the PCs from PCA are chosen for color display. From a feature extraction point of view, PCA's major problem is that some major PCs may not contain more information than minor PCs due to the fact that the ranking criterion for PCs is variance, which can be contributed from both signals and noise. When the data are noisy, the contribution to variance from small objects may be even smaller than that from noise. Thus, the resulting color display constructed from the major PCs may not always include useful information in the original data.

\section{B. NAPCA}

NAPCA's method is to rank PCs in terms of SNR. Assume that $\Sigma$ can be decomposed into signal and noise components, i.e., $\Sigma=\Sigma_{s}+\Sigma_{n}$. NAPCA is performed in two steps [10]. The first step conducts noise whitening to the original data, and the second step performs ordinary PCA to the noise-whitened data. Because the noise variance is unity in the noise-whitened data, the resultant PCs are in the order of SNR. Let $\mathbf{F}$ be the noise-whitening matrix that makes $\Sigma_{n}$ an identity matrix. Transforming $\Sigma$ by $\mathbf{F}$, i.e.,

$$
\mathbf{F}^{T} \boldsymbol{\Sigma} \mathbf{F}=\boldsymbol{\Sigma}_{n \_a d j}
$$

results in $\Sigma_{n \_a d j}$, the covariance matrix with noise being whitened. After finding matrix $\mathbf{G}$ to whiten $\Sigma_{n \_a d j}$, the operator for NAPCA can be constructed as follows:

$$
\mathbf{z}_{N A P C A}=\mathbf{G}^{T} \mathbf{F}^{T}(\mathbf{z}-\mathbf{m}) .
$$

The noise covariance matrix $\boldsymbol{\Sigma}_{n}$ can be estimated using the method in [13]. The major PCs from NAPCA generally contain more object information than those from PCA.

\section{INAPCA}

When we know the object information that needs to be preserved, INAPCA is a more appropriate transform [11]. Here, interference is considered as unwanted signals, such as background clutters. Let $\boldsymbol{\Sigma}=\boldsymbol{\Sigma}_{s}+\boldsymbol{\Sigma}_{i+n}$, where $\boldsymbol{\Sigma}_{i+n}$ is the interference-noise covariance matrix, which contains the second-order statistical information of interference and noise only. Then a matrix $\mathbf{A}$ is determined via eigendecomposition of $\boldsymbol{\Sigma}_{i+n}$ to whiten $\boldsymbol{\Sigma}_{i+n}$, which is used to transform the data as

$$
\mathbf{A}^{T} \boldsymbol{\Sigma} \mathbf{A}=\boldsymbol{\Sigma}_{i+n \_a d j} .
$$

Here, $\Sigma_{i+n \_a d j}$ is the covariance matrix with interference and noise being whitened. If the resultant data are transformed by ordinary PCA, the net effect is to order PCs in terms of signalto-interference-plus-noise ratio (SINR). Following the same idea of NAPCA, the matrix $\mathbf{E}$ can be found via eigendecomposition of $\Sigma_{i+n \_a d j}$ to whiten $\Sigma_{i+n \_a d j}$. Then the PC images using INAPCA can be computed by

$$
\mathbf{z}_{I N A P C A}=\mathbf{E}^{T} \mathbf{A}^{T}(\mathbf{z}-\mathbf{m}) .
$$

In general, the interference information is difficult to obtain. Fortunately, $\boldsymbol{\Sigma}_{i+n}$ can easily be computed via orthogonal subspace projection (OSP) without the estimation of interference and noise. Let the desired signature matrix be denoted as $\mathbf{S}=$ $\left\lfloor\mathbf{s}_{1} \mathbf{s}_{2} \cdots \mathbf{s}_{p}\right\rfloor$ with $p$ signatures or classes. To get a data set with interference and noise only, all these $p$ signatures are annihilated by projecting the original data onto the subspace that is orthogonal to these desired signatures using the following OSP operator [14]:

$$
\mathbf{P}^{\perp}=\mathbf{I}-\mathbf{S}\left(\mathbf{S}^{T} \mathbf{S}\right)^{-1} \mathbf{S}^{T}
$$

where $\mathbf{I}$ is an identity matrix. Let the projected data be denoted as $\hat{\mathbf{z}}$, where only interference and noise is present. Then $\boldsymbol{\Sigma}_{i+n}$ can be estimated as the sample covariance matrix of $\hat{\mathbf{z}}$.

\section{ICA}

ICA is a frequently used unsupervised classification method [15]. Its basic idea is to decompose a set of multivariate signals into a base of statistically independent sources with minimal loss of information content. Quite a few ICA algorithms exist. 
The following is the rationale of the well-known FastICA algorithm with a kurtosis maximization criterion [16].

Let $\mathbf{Z}=\left[\mathbf{z}_{1} \mathbf{z}_{2} \cdots \mathbf{z}_{N}\right]$ be an $L \times N$ data matrix with $N L$-dimensional pixels. Also let $\mathbf{w}$ be the desired projector, and let $\mathbf{y}=\left(y_{1} y_{2} \cdots y_{N}\right)$ be the projected data (after mean removal and data whitening). Denote $F(\bullet)$ as a function measuring independency. For instance, $F(y)$ can measure the kurtosis $\kappa(y)$ of the projected data, i.e.,

$$
F(y)=\kappa(y)=E\left[(y)^{4}\right]-3 .
$$

Then the task is to find an optimal w such that $\kappa(y)$ is maximal. The objective function for this optimization problem is

$$
J(\mathbf{w})=\max _{\mathbf{w}}\{\kappa(y)\}=\max _{\mathbf{w}}\left\{\kappa\left(\mathbf{w}^{T} \mathbf{z}\right)\right\} .
$$

Taking the derivative with respect to $\mathrm{w}$ yields

$$
\Delta \mathbf{w}=\frac{\partial \kappa}{\partial \mathbf{w}}=4 E\left(y^{3} \mathbf{z}\right)
$$

Then the gradient-descent or fixed-point adaptation can be used to determine $\mathbf{w}$. After the first $\mathbf{w}$, denoted as $\mathbf{w}_{1}$, is found, it is used to transform the data for the first classification map. To find a second $\mathbf{w}$, denoted as $\mathbf{w}_{2}$, for another class, data matrix $\mathbf{Z}$ is projected onto the orthogonal subspace of $\mathbf{w}_{1}$ before searching $\mathbf{w}_{2}$. The algorithm continues until all the classes are classified.

\section{E. $L D A$}

Fisher's LDA is a standard technique for dimension reduction in pattern recognition. Assume that there are $k$ training sample vectors given by $\left\{\mathbf{z}_{i}\right\}_{i=1}^{k}$ for $p$-classes: $\theta_{1}, \theta_{2}, \cdots, \theta_{p}$ and that there are $k_{j}$ samples for the $j$ th class. Let $\mu$ be the mean of the entire training samples and $\mu_{j}$ be the mean of the $j$ th class. The LDA projects the original high-dimensional data onto a lowdimensional space, where all the classes are well separated by maximizing the Raleigh quotient, i.e., the ratio of between-class scatter matrix $\mathbf{S}_{W}$ to within-class scatter matrix $\mathbf{S}_{B}$, which are defined as [17]

$$
\begin{aligned}
\mathbf{S}_{W} & =\sum_{\mathbf{z}_{i} \in \theta_{j}}\left(\mathbf{z}_{i}-\boldsymbol{\mu}_{j}\right)\left(\mathbf{z}_{i}-\boldsymbol{\mu}_{j}\right)^{T} \\
\mathbf{S}_{B} & =\sum_{j=1}^{p} k_{j}\left(\boldsymbol{\mu}_{j}-\boldsymbol{\mu}\right)\left(\boldsymbol{\mu}_{j}-\boldsymbol{\mu}\right)^{T}
\end{aligned}
$$

respectively. The $\mathbf{w}$ maximizing the Raleigh quotient can be determined by solving the following generalized eigenproblem:

$$
\mathbf{S}_{B} \mathbf{w}=\lambda \mathbf{S}_{W} \mathbf{w}
$$

where $\lambda$ is a generalized eigenvalue. Because the rank of $\mathbf{S}_{B}$ is $p-1$, there are $p-1$ eigenvectors associated with $p-1$ nonzero eigenvalues. Therefore, an $L \times(p-1)$ matrix $\mathbf{W}$ can be found to transform the original $L$-dimensional data into a $(p-1)$-dimensional space where the $p$ classes can be well separated.

\section{F. CLDA}

Fisher's LDA performs dimension reduction only. To achieve classification simultaneously with the transformation, a CLDA was proposed by imposing the constraint that the different classes were aligned along different directions as in [18]

$$
\mathbf{w}_{l}^{T} \boldsymbol{\mu}_{j}=\delta_{l j} \text { for } 1 \leq l, j \leq p .
$$

The resulting classifier was the OSP after data whitening. Let the transform matrix be denoted as $\mathbf{W}=\left[\mathbf{w}_{1}, \mathbf{w}_{2}, \cdots, \mathbf{w}_{p}\right]$. It was proven that $\mathbf{W}$ is equivalent to the following operator [19]:

$$
\mathbf{W}_{C L D A}=\boldsymbol{\Sigma}^{-1} \mathbf{S}\left(\mathbf{S}^{T} \boldsymbol{\Sigma}^{-1} \mathbf{S}\right)^{-1}
$$

where $\mathbf{S}$ contains the $p$-class signatures to be classified.

\section{G. $U F C L U$}

Linear mixture analysis is a widely used method to classify and quantify endmember materials in hyperspectral imagery. With a slight abuse of notation, let the number of endmembers be $p$ and their signature matrix be $\mathbf{S}$. According to the linear mixture model (LMM), a pixel $\mathbf{z}$ can be represented as

$$
\mathbf{z}=\mathbf{S} \boldsymbol{\alpha}+\mathbf{n}
$$

where $\boldsymbol{\alpha}=\left(\alpha_{1} \cdots \alpha_{i} \cdots \alpha_{p}\right)^{T}$ is a $p \times 1$ column vector called the abundance vector, whose $i$ th element represents the proportion of the $i$ th endmember material in the pixel $\mathbf{z}$. Here, $\mathbf{n}$ represents an additive noise or measurement error.

Because $\alpha_{i}$ represents abundance, it should be a nonnegative value. Also, the pixel is assumed to be a linear combination of all the endmembers. Thus the sum of the abundances should be one. These two constraints can be formed as follows:

$$
\sum_{i=1}^{p} \alpha_{i}=1 \text { and } 0 \leq \alpha_{i} \leq 1 \text { for } 1 \leq i \leq p .
$$

When $\mathbf{S}$ is known, a constrained optimization process, such as FCLU, can be imposed to estimate the $\boldsymbol{\alpha}$ that yields the minimum estimation error when the constraints in (17) are satisfied. It can be simply achieved via quadratic programming. If $\mathbf{S}$ is unknown, then UFCLU needs to be performed [20].

When the number of endmembers is unknown, a large number can be assumed first to run the UFCLU algorithm. Then similar endmember signatures can be combined after similarity comparison using a spectral angle mapper (SAM) [21]. Endmember signatures corresponding to noisy abundance images with large entropies are removed. The remaining signatures are used in the supervised FCLU to generate the final abundance images for color display.

Note that the performance of supervised FCLU strongly relies on the accuracy of the number of endmembers and their signatures. If this information is incorrect, the results can be misleading, even if the constraints in (17) are imposed [22]. This can be a problem due to the complexity of real image scenes and the in-field spectral variability. Thus, we only study UFCLU in this research. 
TABLE I

SUMMARY OF THE NINE COLOR DISPLAY SCHEMES IN THIS PAPER

\begin{tabular}{|c|c|c|c|}
\hline & Nature & Processing & \# Display Elements \\
\hline CLDA & Supervised & Classification & Number of classes \\
\hline LDA & Supervised & Transformation & Number of classes -1 \\
\hline INAPCA & Supervised & Transformation & User defined \\
\hline ICA & Unsupervised & Classification & User defined \\
\hline UFCLU & Unsupervised & Classification & User defined \\
\hline NAPCA & Unsupervised & Transformation & User defined \\
\hline PCA & Unsupervised & Transformation & User defined \\
\hline CMF & Unsupervised & None & 3 \\
\hline TBC & Unsupervised & None & 3 \\
\hline
\end{tabular}

\section{H. Technique Summary}

The characteristics of the aforementioned techniques along with TBC and CMF are summarized in Table I. CLDA, LDA, and INAPCA are supervised methods, which means that the class signatures are known a priori; the rest of techniques are unsupervervised methods. CLDA, ICA, and UFCLU are classification methods, whereas LDA, INAPCA, NAPCA, and PCA are transformation methods. CMF and TBC do not include a data processing step, although CMF does include a transformation step to directly produce a color display but without information transfer, as in typical data processing.

After classification maps or transformed components, called display elements (DE) hereafter, are available, they will be used to generate a color display. As shown in Table I, the numbers of DEs from the supervised CLDA and LDA are directly related to the number of classes $p$, there are three classes for CMF and TBC, and others are user defined.

Note that the aforementioned classifiers produce soft classification results, which are directly used for visualization, due to the dominant presence of mixed pixels in remotely sensed imagery.

\section{Color Display Strategies}

Assume that $q$ DEs are used to generate a color display. In general, $q$ and $p$ are not equal; they are equal when a supervised classification result is used for visualization. The detailed steps for generating a color display include color selection, color assignment, display element adjustment, and color display reproduction.

\section{A. Perception-Based Distinctive Color Selection}

In approximately uniform color spaces, such as CIELAB and CIELUV, the perceptual distance between two colors is roughly proportional to the Euclidean distance between the corresponding points in the color space [23], [24]. Healey addressed three criteria for color selection: color category, color distance, and linear separation [25]. When there are fewer than 10 colors to be selected, the color labels selected from a uniform color space can belong to different color categories [25], [26].

The Munsell color space is designed to be perceptually uniform. Developed by Albert H. Munsell in 1905, the Munsell color model uses three dimensions: hue, value, and chroma to specify a color. Hue is represented by a circular band divided

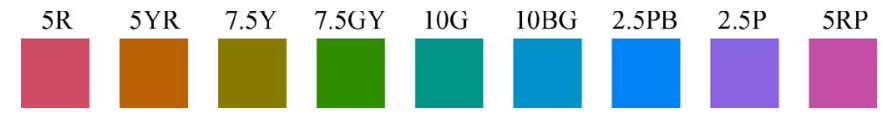

Fig. 1. Nine maximally distinct colors selected using the Munsell color tables.

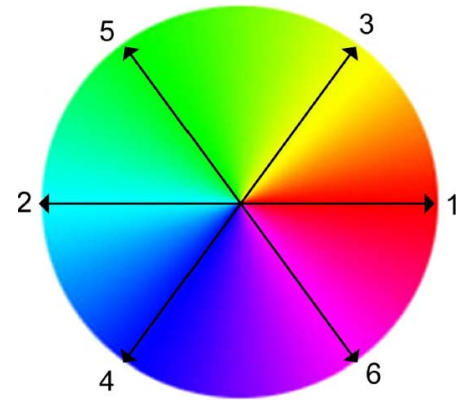

Fig. 2. Six classes are assigned distinctive colors according to their similarity.

into 10 sections: red, yellow-red, yellow, green-yellow, green, blue-green, blue, purple-blue, purple, and red-purple, denoted as R, YR, Y, GY, G, BG, B, PB, P, and RP, respectively. Each section can be further divided into 10 subsections for finer divisions of hue. Value represents the lightness or darkness of a color, and it is divided into 11 sections numbered 0 through 10. Chroma defines the strength or weakness of a color, and it is measured in numbered steps starting at 1 . The format "hue value/chroma" is employed to specify a Munsell color, and a number preceding the hue defines the subsection. For instance, 5R5/5 denotes a strong red. Munsell colors can be perceptually "balanced," because hues directly opposite one another will be balanced if the value and chroma are equal. For instance, $5 \mathrm{R} 5 / 5$ is perceptually balanced with $5 \mathrm{BG} 5 / 5$. In addition to approximate perceptual uniformity and convenient balance, Munsell colors can be easily displayed and printed. They are associated with the Inter-Society Color Council and the National Bureau of Standards (ISCC-NBS) standard color naming system [27]. Wyszecki and Stiles provided $(X, Y, Z)$ values for many Munsell colors [24].

Therefore, Munsell color tables are used for distinctive color selection in this research. Fig. 1 shows the selected colors (with the same value 5 and chroma 12) if nine colors are needed.

\section{B. Color Assignment Considering Display Element Similarity}

To improve class separability in the final color display image, it is intuitive to assign very different colors to similar classes. This can be easily accomplished for a supervised classification method, such as CLDA. Let class signatures be arranged in a sequence based on their signature similarity using SAM. Without loss of generality, $\mathbf{s}_{i}$ is more similar to $\mathbf{s}_{i+1}$ than to $\mathbf{s}_{i+2}$ after this arrangement. Then the color for the $i$ th classification map corresponding to $\mathbf{s}_{i}$ is less similar to the $(i+1)$ th map than to the $(i+2)$ th. An example of the resultant interleaving color assignment strategy is illustrated in Fig. 2.

For an unsupervised classification method, such as ICA and UFCLU, or a transformation method, such as LDA, colors can be assigned based on the spatial similarity between the DEs. The similarity metric is correlation coefficient (CC). Without loss of generality, assume that DEs are arranged according to 
CC, i.e., $\mathrm{DE}_{i}$ is more similar to $\mathrm{DE}_{i+1}$ than to $\mathrm{DE}_{i+2}$. Then the color assigned to $\mathrm{DE}_{i}$ should be less similar to $\mathrm{DE}_{i+1}$ than that to $\mathrm{DE}_{i+2}$, illustrated in Fig. 2.

In some transformation methods, such as PCA, NAPCA, and INAPCA, the transformed components are uncorrelated. Thus all cross-component CCs are zero. In this case, each image can be divided into nonoverlapping blocks, and the $\mathrm{CC}$ between two corresponding blocks at the same location in two different DEs is computed. Then the similarity between each pair of DEs can be arranged in terms of the average block CCs.

\section{Display Element Adjustment}

In some techniques, e.g., PCA, ICA, and LDA, the solutions may be sign indeterminable. In this case, it needs to be decided if the generated or negative classification map/transformed components should be used as a DE. To make the adjustment automatic, the maximum positive value $b_{\max }$ and minimum negative value $b_{\min }$ are determined among all the pixels in a DE. If the absolute value of $b_{\min }$ is greater than $b_{\max }$, i.e., $\left|b_{\min }\right|>b_{\max }$, this indicates that the negative image should be used as a DE.

If the range of pixel values in one DE is much larger than in others, then this DE will dominate the final color display. Therefore, each DE needs to be normalized.

As for the number of DEs to be used for color display, it should be less than ten to make sure the colors assigned for DEs are from different color categories [25], [26]. When there are more than 10 classes present, similar classes have to be merged based on CC or SAM.

\section{Color Display Reproduction}

Let the number of DEs for the final color display be $q$. For the $i$ th DE, a $3 \times 1$ color vector, denoted as $\mathbf{c}_{i}=\left(r_{i} g_{i} b_{i}\right)^{T}$, is assigned using the criteria for color assignment presented in Sections III-A and B. Let the $q$ DEs after adjustment form an $N \times q$ data matrix denoted as $\mathbf{B}=\left[\mathbf{b}_{1}, \mathbf{b}_{2}, \cdots, \mathbf{b}_{q}\right]$, where $\mathbf{b}_{i}$ is the vector formed by stacking the columns of $\mathrm{DE}_{i}$ (i.e., arranging the $i$ th 2-D classification map or transformed component into a 1-D vector). Then, a color matrix of size $3 \times q$ is constructed as $\mathbf{C}=\left[\mathbf{c}_{1} \cdots \mathbf{c}_{i} \cdots \mathbf{c}_{q}\right]$. The final color display is created by

$$
\mathbf{D}=\mathbf{C B}^{T}
$$

where $\mathbf{D}$ is the three-channel image with $N$ pixels. The values in each channel of $\mathbf{D}$ need to be normalized into [0 1 1] before an actual RGB display. Equation (18) is also called chromatic mixing.

\section{E. Evaluation on Visual Interpretation}

The metrics to be used in the evaluation of visual products are objective-dependent. For example, in [12] where the visualizations were not task specific, the $\mathrm{CC}$ between the pixel spectral distances in an original hyperspectral data and the corresponding perceptual color distances in a color display

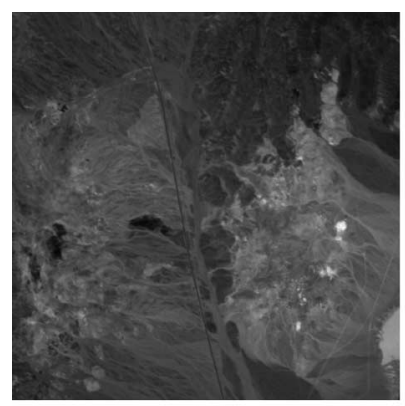

(a)

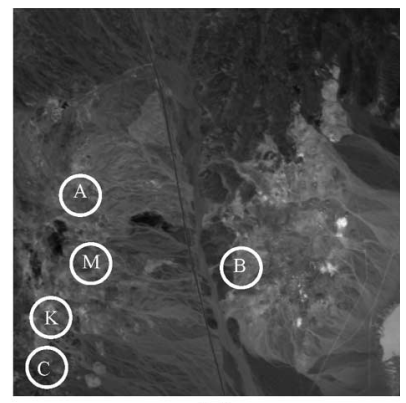

(b)
Fig. 3. AVIRIS Cuprite image scene. (a) Band 100 and (b) spatial location of five minerals: alunite (A), buddingtonite $(B)$, calcite $(C)$, kaolinite $(\mathrm{K})$, and muscovite (M).

are measured; a greater $\mathrm{CC}$ means a higher effectiveness in maintaining the original spectral difference. In this paper, the objective is class separability, and thus we evaluate the color distance between class centers (i.e., the Euclidean distance $\Delta E$ between the color vectors corresponding to class centers) in an approximately perceptually uniform color space (e.g., CIELUV), and a larger average distance among class centers generally means better perceptual separation. In addition, class separability in a produced color image can also be evaluated by applying a statistical detector or classifier. Here, a detection algorithm called constrained energy minimization (CEM) is employed [28] to generate a gray scale detection map for each class. When the ground truth is unavailable, each map is compared with its counterpart from using all the original bands. If they are similar (using the $\mathrm{CC}$ as spatial similarity metric), this means that the color display is satisfactory in preserving and discriminating different classes.

\section{EXPERIMENT}

The data used in the experiment are the Airborne Visible/ Infrared Imaging Spectrometer (AVIRIS) Cuprite subimage scene sized $350 \times 350$ shown in Fig. 3, which were taken over Cuprite, NV, in 1997. After water absorption and lowSNR bands were removed, 189 bands were left. This scene has been well studied mineralogically [29]. It is known that at least five minerals were present: alunite (A), buddingtonite (B), calcite $(\mathrm{C})$, kaolinite $(\mathrm{K})$, and muscovite $(\mathrm{M})$. Their approximate spatial locations are marked in Fig. 3(b). Fig. 4 presents the CLDA classification results where the spatial distributions of these five materials are elucidated so that they can be used as "road maps" for visualization. According to our objective, the generated color display should maximize this five-class separability. Note that there may be more than five foreground materials, and the background information (i.e., the number of background materials and their signatures) is unknown, which is a typical situation for remote sensing data.

Fig. 5(a)-(g) shows the color displays using the data processing techniques described in Section II. In the three supervised approaches-CLDA, LDA, and INAPCA - the five material signatures were used for classification or transformation. In the other unsupervised approaches, no prior information about the image scene was assumed. When using INAPCA, ICA, 


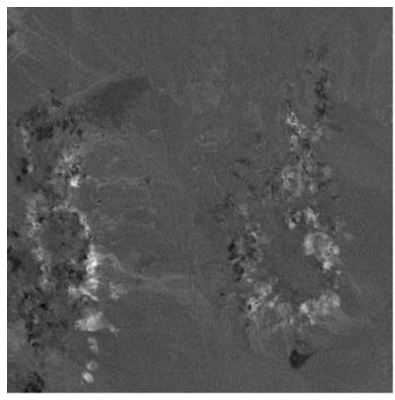

(a)

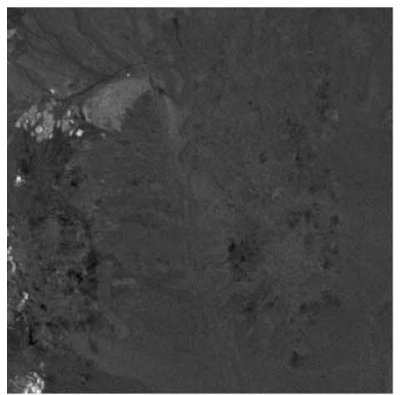

(c)

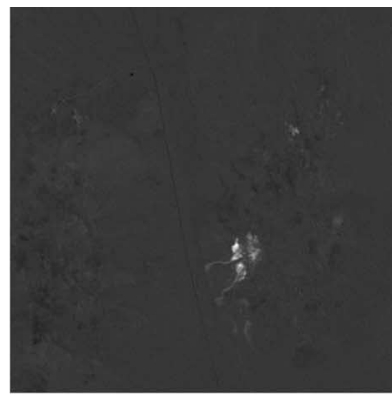

(b)

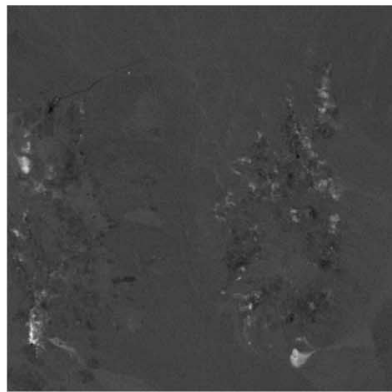

(d)

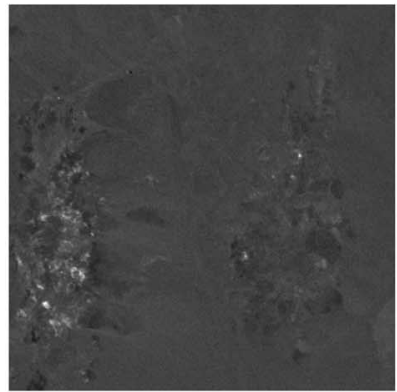

(e)

Fig. 4. CLDA classification of the AVIRIS Cuprite image scene. (a) Alunite (A), (b) buddingtonite (B), (c) calcite (C), (d) kaolinite (K), and (e) muscovite (M).

UFCLU, NAPCA, and PCA, the number of DEs for color display is defined by users. In this experiment, nine DEs were involved in color display generation. The Munsell color tables were employed to assign distinctive colors to DEs. As mentioned in Section III-B, the similarity between DEs was considered when assigning colors.

For comparison purposes, the CMF and TBC methods were also applied. In the CMF method, the Commission Internationale d'Eclairage (CIE) 1964 tristimulus color matching functions were adopted, from which the three 189-dimensional discrete weighting functions were used to generate the linear integrations for the red, green, and blue channels. In the TBC method, three distinct bands in green, red, and NIR spectral region were selected based on entropy, and they were assigned to the three-color channels, respectively. Thus, the TBC color display is very similar to the CMF color display as shown in Fig. 5(h) and (i). Compared to the color displays from the data processing results, the color displays from CMF and TBC obviously provide less information about the distribution of the five desired classes. For instance, on the right side of the CMF and TBC visualizations shown in Fig. 5, it is difficult

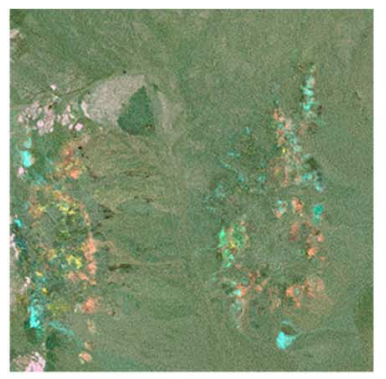

(a)

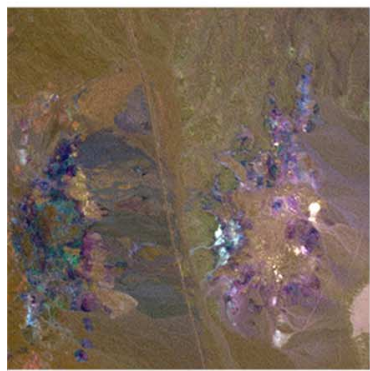

(c)

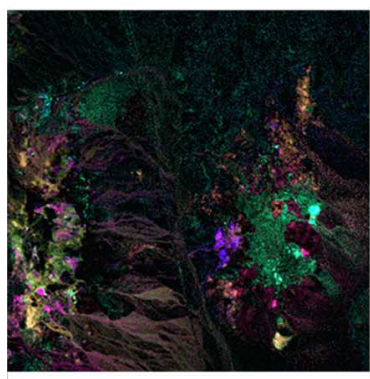

(e)

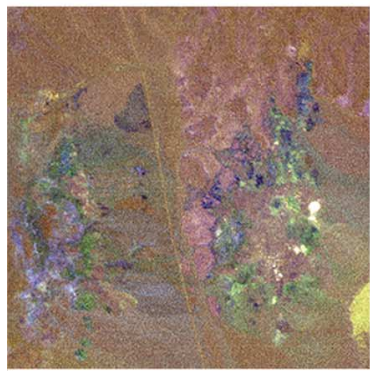

(g)

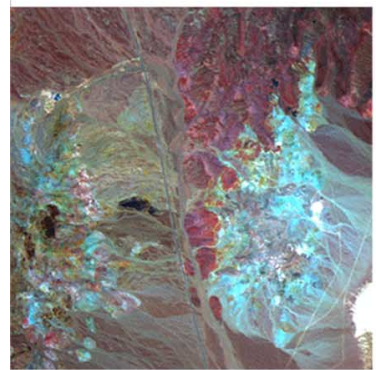

(i)

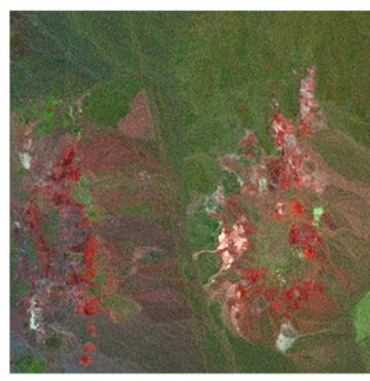

(b)

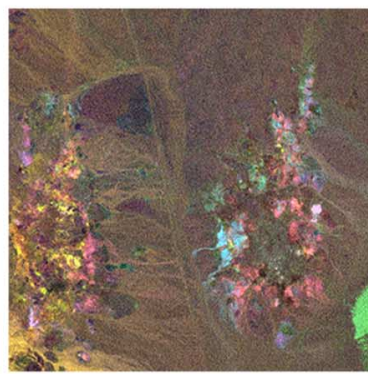

(d)

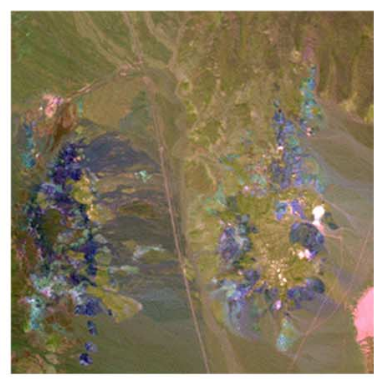

(f)

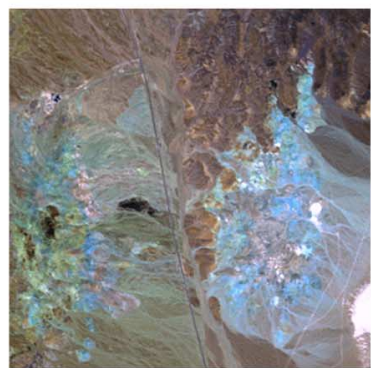

(h)
Fig. 5. Color display using different methods. (a) CLDA. (b) LDA (c) INAPCA. (d) ICA. (e) UFCLU. (f) NAPCA. (g) PCA. (h) CMF. (i) TBC.

to differentiate the regions with high probability of alunite, buddingtonite, and kaolinite, which are displayed in blue.

Fig. 5(c), (f), and (g) were generated using INAPCA, NAPCA, and PCA, respectively. The PCA-generated color 
TABLE II

Evaluation Using Color Distance in the CiElUV Space $\Delta E=$ Average EuClidean Distance BeTWEen Class CENTERS IN THE CIELUV SPACE

\begin{tabular}{|c|c|}
\hline & $\Delta \mathrm{E}$ \\
\hline CLDA & 65.48 \\
\hline LDA & 57.20 \\
\hline INAPCA & 70.76 \\
\hline ICA & 72.27 \\
\hline UFCLU & 97.93 \\
\hline NAPCA & 43.58 \\
\hline PCA & 35.80 \\
\hline CMF & 22.02 \\
\hline TBC & 31.02 \\
\hline
\end{tabular}

$\Delta \mathrm{E}=$ Average Euclidean distance between class centers in the CIELUV space.

display is noisier than the other two, because the PCs were not ranked in terms of image quality, and some high-ranked PCs (e.g., the sixth PC) contained much noise. NAPCA displays the minerals with higher contrast. The NAPCA-generated color display contains more class information than the one from PCA. For example, kaolinite can be more easily distinguished from alunite on the left side of the image because the former was displayed in green-blue and the latter in purple. However, both the PCA- and NAPCA-generated color displays present less class information that the one generated by INAPCA. For instance, buddingtonite is submerged in the background in Fig. 5(f) and (g), but it is distinctively displayed in light blue in Fig. 5(c). This is because INAPCA performs supervised transformation, whereas PCA and NAPCA conduct unsupervised transformation.

The CLDA-generated color display clearly presents the five classified materials in Fig. 5(a); they are shown in orange, light green, light magenta, light blue, and yellow, respectively. Fig. 5(b) is the color display from the LDA transform, where alunite, buddingtonite, calcite, and kaolinite are visible, but muscovite is not. This may be because the LDA transform results in only four components in this case, and there are not enough dimensions to accommodate all the materials. The display in Fig. 5(d) from the ICA classification maps looks very colorful, where all the materials can be visually detected except calcite. The UFCLU-generated color display in Fig. 5(e) has the highest contrast.

To quantitatively evaluate the color displays in Fig. 5, the Euclidean distance between each pair of materials in the CIELUV space was calculated and averaged. As shown in Table II, the UFCLU-generated color display has the largest color distance because of the highest contrast; CLDA, LDA, INAPCA, and ICA have large color distances; PCA, CMF, and TBC have the smallest color distances.

To objectively assess the material information preserved in the color display images and how to separate them easily, the CEM detector was applied to the original data and color displays. As listed in Table III, the CC was used to compare the similarity between the corresponding detection maps. We can see that CLDA and INAPCA contain most of the mater-
TABLE III

Evaluation Using CEM (CORRELATION COEFFICIENT)

\begin{tabular}{|c|c|c|c|c|c|c|}
\hline & A & B & C & K & M & Ave. \\
\hline CLDA & 0.79 & 0.74 & 0.64 & 0.67 & 0.63 & $\mathbf{0 . 6 9}$ \\
\hline LDA & 0.57 & 0.55 & 0.27 & 0.63 & 0.11 & $\mathbf{0 . 4 3}$ \\
\hline INAPCA & 0.67 & 0.63 & 0.45 & 0.54 & 0.54 & $\mathbf{0 . 5 7}$ \\
\hline ICA & 0.61 & 0.58 & 0.07 & 0.44 & 0.53 & $\mathbf{0 . 4 4}$ \\
\hline UFCLU & 0.17 & 0.51 & 0.17 & 0.63 & 0.33 & $\mathbf{0 . 3 6}$ \\
\hline NAPCA & 0.56 & 0.12 & 0.16 & 0.51 & 0.37 & $\mathbf{0 . 3 4}$ \\
\hline PCA & 0.50 & 0.15 & 0.16 & 0.19 & 0.09 & $\mathbf{0 . 2 2}$ \\
\hline CMF & 0.53 & 0.13 & 0.14 & 0.26 & 0.10 & $\mathbf{0 . 2 3}$ \\
\hline TBC & 0.41 & 0.11 & 0.10 & 0.37 & 0.21 & $\mathbf{0 . 2 4}$ \\
\hline
\end{tabular}

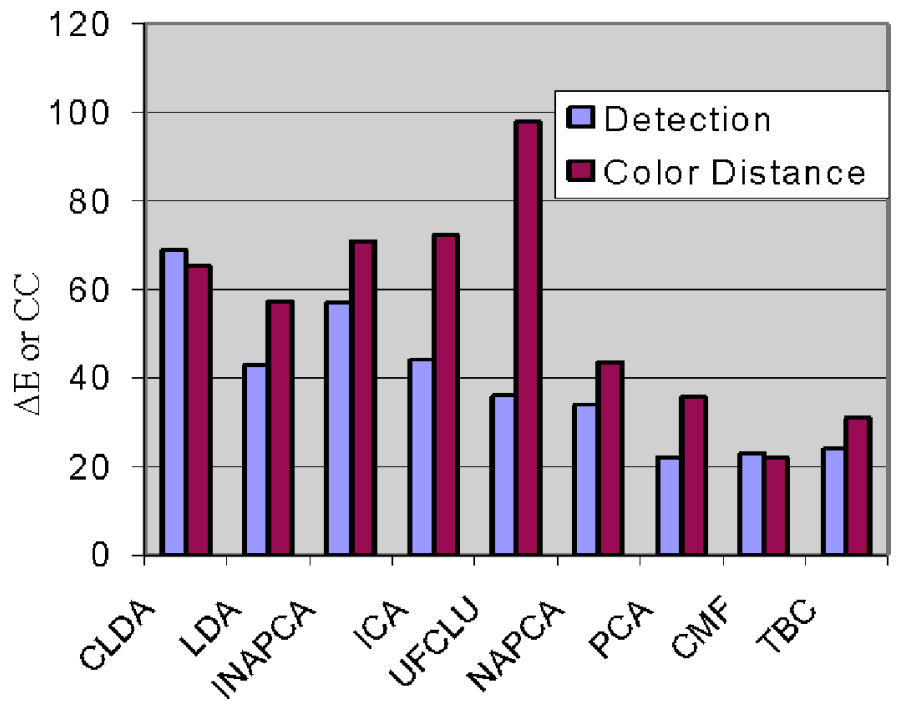

Fig. 6. Chart for performance comparison using the results in Tables II and III (the CCs for detection are multiplied by 100 for visualization purposes).

ial information, whereas PCA, CMF, and TBC are the worst in terms of class separation. UFCLU is not among the best. This may be because the color display has very high contrast, which makes the detection maps look very different from those produced from the original data.

Fig. 6 summarizes the results in Tables II and III. We can also see that the order of color distances does not precisely match the performance order in detection. The largest discrepancy appears in the UFCLU method, and second largest in the ICA method. Overall, however, the quantified detection performance is in accordance with the perceptual color distance, i.e., a color display providing better detection and discrimination generally has larger color distance between different classes. The discrepancy mainly comes from the nonlinearity of human visual perception and the linear nature of the CEM detector.

To further investigate the impact of the number of DEs used for color display, the three PCA-class techniques were studied by choosing different number of PCs. Table IV lists the color distance and detection $\mathrm{CC}$ in various cases. We can see that using more PCs does not guarantee better performance. This is because the color combination may become very complicated if the number of DEs is too large, making class separation more difficult. In this experiment, the color display from nine PCs was better than the one from ten PCs in PCA, NAPCA, and INAPCA. For PCA, the use of the first three PCs generated the 
TABLE IV

COMPARING THE USE OF A DIFFERENT NUMBER OF PCS

\begin{tabular}{|c|c|c|}
\hline & CC for Detection & $\Delta \mathrm{E}$ \\
\hline PCA 3PCs & 0.25 & 38.28 \\
\hline PCA 6PCs & 0.17 & 30.25 \\
\hline PCA 9 PCs & 0.22 & 35.80 \\
\hline PCA 10PCs & 0.16 & 35.70 \\
\hline NAPCA 3PCs & 0.23 & 40.12 \\
\hline NAPCA 6PCs & 0.25 & 46.15 \\
\hline NAPCA 9PCs & 0.34 & 43.58 \\
\hline NAPCA 10PCs & 0.31 & 36.88 \\
\hline INAPCA 3PCs & 0.25 & 42.29 \\
\hline INAPCA 6PCs & 0.47 & 65.43 \\
\hline INAPCA 9PCs & 0.57 & 70.76 \\
\hline INAPCA 10PCs & 0.55 & 56.27 \\
\hline
\end{tabular}

$\mathrm{CC}=$ Spatial correlation coefficient;

$\Delta \mathrm{E}=$ Average Euclidean distance between class centers in the CIELUV space.

best results. In addition to the problem from color combination, another reason is that some PCs (after the first three PCs) had low quality, and adding them resulted in performance degradation. For NAPCA and INAPCA, using more PCs generally can generate better results, provided that there are fewer than ten PCs. When there were ten PCs used as DEs, then the performance began to deteriorate. This tells us that the number of DEs cannot be too large even when the DEs have good quality.

\section{CONCLUSION}

We investigated the color display of hyperspectral data with a single RGB composite. We focused on the impact of data processing (i.e., transformation for information compact, and classification) on information preservation and class separability. Six techniques were proposed: supervised CLDA, LDA, and INAPCA and unsupervised ICA, UFCLU, and NAPCA. They were compared with the three existing color display techniques in the literature: PCA, CMF, and TBC. Based on the experimental results, the following conclusions can be drawn, which are suitable to other data transformation and classification approaches for color display generation in addition to the nine popular techniques investigated here.

1) Processing the data can produce better color displays, whereas using the classification approaches generally is better than using the transformation approaches in terms of higher class separability in the produced color display with concomitant complex implementation.

2) When desired class information is known, a supervised approach can be applied. CLDA is the supervised method providing the best result. When no prior information is available, an unsupervised technique has to be applied. ICA is the unsupervised method providing the best result.

3) In the unsupervised situation, a transformation-based approach is easier to implement than a classification-based approach. For instance, both ICA and UFCLU need to generate many classification maps to separate different classes, but too many classification maps may split the same class into several subclasses which need a post- processing step for class merging. In most cases, a simple transformation technique such as NAPCA may be the alternative.

4) $\mathrm{TBC}$ produces a color display similar to $\mathrm{CMF}$ when a green band is assigned red, a red band is assigned green, and a NIR band is assigned blue. Both can provide the property of consistent rendering but with very low class separability.

5) This research is focused on colorimetry only, providing a quick and simple overview of a scene. The number of classification maps or transformed components for color combination is constrained to be small, say, less than ten; otherwise, color differentiation in the final display becomes very difficult. For a complex image scene with many classes, more advanced visualization techniques need to be adopted, such as the one in [30].

\section{ACKNOWLEDGMENT}

The authors would like to thank Drs. Chein-I Chang and Antonio Plaza for providing the data and ground truth used in the experiment.

\section{REFERENCES}

[1] J. Bryant, "On displaying multispectral imagery," Photogramm. Eng. Remote Sens., vol. 54, no. 12, pp. 1739-1743, Dec. 1988.

[2] L. Di and D. C. Rundquist, "Color-composite image generation on an eight-bit graphics workstation," Photogramm. Eng. Remote Sens., vol. 54, no. 12, pp. 1745-1748, Dec. 1988.

[3] J. M. Durand and Y. H. Kerr, "An improved decorrelation method for the efficient display of multispectral data," IEEE Trans. Geosci. Remote Sens., vol. 27, no. 5, pp. 611-619, May 1989.

[4] Q. Lin and J. Allebach, "Displaying multispectral images on video terminals in RGB color," in Proc. IEEE Int. Geosci. Remote Sens. Symp., 1990, pp. $357-360$.

[5] M. Beauchemin and K. B. Fung, "On statistical band selection for image visualization," Photogramm. Eng. Remote Sens., vol. 67, no. 5, pp. 571574, 2001.

[6] J. S. Tyo, A. Konsolakis, D. I. Diersen, and R. C. Olsen, "Principalcomponents-based display strategy for spectral imagery," IEEE Trans. Geosci. Remote Sens., vol. 41, no. 3, pp. 708-718, Mar. 2003.

[7] V. Tsagaris and V. Anastassopoulos, "Multispectral image fusion for improved RGB representation based on perceptual attributes," Int. J. Remote Sens., vol. 26, no. 15, pp. 3241-3254, Aug. 2005.

[8] V. Tsagaris, V. Anastassopoulos, and G. A. Lampropoulos, "Fusion of hyperspectral data using segmented PCT for color representation and classification," IEEE Trans. Geosci. Remote Sens., vol. 43, no. 10, pp. 23652375, Oct. 2005.

[9] A. A. Green, M. Berman, P. Switzer, and M. D. Craig, "A transformation for ordering multispectral data in terms of image quality with implications for noise removal," IEEE Trans. Geosci. Remote Sens., vol. 26, no. 1, pp. 65-74, Jan. 1988.

[10] R. E. Roger, "A faster way to compute the noise-adjusted principal components transform matrix," IEEE Trans. Geosci. Remote Sens., vol. 32, no. 6, pp. 1194-1196, Jun. 1994.

[11] C.-I Chang and Q. Du, "Interference and noise adjusted principal components analysis," IEEE Trans. Geosci. Remote Sens., vol. 37, no. 9, pp. 2387-2396, Sep. 1999.

[12] N. P. Jacobson and M. R. Gupta, "Design goals and solutions for display of hyperspectral images," IEEE Trans. Geosci. Remote Sens., vol. 43, no. 11, pp. 2684-2693, Nov. 2005.

[13] R. E. Roger and J. F. Arnold, "Reliably estimating the noise in AVIRIS hyperspectral imagers," Int. J. Remote Sens., vol. 17, no. 10, pp. 19511962, 1996.

[14] J. C. Harsanyi and C.-I Chang, "Hyperspectral image classification and dimensionality reduction: An orthogonal subspace projection," IEEE Trans. Geosci. Remote Sens., vol. 32, no. 4, pp. 779-785, Apr. 1994. 
[15] A. Hyvärinen, J. Karhunen, and E. Oja, Independent Component Analysis. New York: Wiley-Interscience, 2001.

[16] A. Hyvärinen, "Fast and robust fixed-point algorithms for independent component analysis," IEEE Trans. Neural Netw., vol. 10, no. 3, pp. 626634, Mar. 1999.

[17] R. O. Duda and P. E. Hart, Pattern Classification and Scene Analysis. New York: Wiley, 1973.

[18] Q. Du and C.-I Chang, "A linear constrained distance-based discriminant analysis for hyperspectral image classification," Pattern Recognit., vol. 34, no. 2, pp. 361-373, Feb. 2001.

[19] Q. Du and H. Ren, "Real-time constrained linear discriminant analysis to target detection and classification in hyperspectral imagery," Pattern Recognit., vol. 36, no. 2, pp. 1-12, Jan. 2003.

[20] D. C. Heinz and C.-I Chang, "Fully constrained least squares linear spectral mixture analysis method for material quantification in hyperspectral imagery," IEEE Trans. Geosci. Remote Sens., vol. 39, no. 3, pp. 529-545, Mar. 2001.

[21] F. A. Kruse et al., "The spectral image processing system (SIPS)-Interactive visualization and analysis of imaging spectrometer data," Remote Sens. Environ., vol. 44, no. 2/3, pp. 145-163, 1993.

[22] D. M. Rogge, B. Rivard, J. Zhang, and J. Feng, "Iterative spectral unmixing for optimizing per-pixel endmember sets," IEEE Trans. Geosci. Remote Sens., vol. 44, no. 12, pp. 3725-3736, Dec. 2006.

[23] P. K. Roberston, "Visualizing color gamuts: A user interface for the effective use of perceptual color spaces in data display," IEEE Comput. Graph. Appl., vol. 8, no. 9, pp. 50-64, Sep. 1988.

[24] G. Wyszecki and W. S. Stiles, Color Science: Concepts and Methods, Quantitative Data and Formulae. New York: Wiley-Interscience, 2000.

[25] C. G. Healey, "Effective visualization of large multidimensional datasets," Ph.D. dissertation, Univ. British Columbia, Vancouver, BC, Canada, 1996.

[26] C. Ware, Information Visualization, 2nd ed. San Mateo, CA: Morgan Kaufmann, 2004.

[27] P. Campadelli, R. Schettini, and S. Zuffi, "A system for the automatic selection of conspicuous color sets for qualitative data display," IEEE Trans. Geosci. Remote Sens., vol. 39, no. 10, pp. 2283-2286, Oct. 2001.

[28] J. C. Harsanyi, "Detection and classification of subpixel spectral signatures in hyperspectral image sequences," Univ. Maryland Baltimore County, Baltimore, MD, 1993.

[29] A. Plaza and C.-I Chang, "Impact of initialization on design of endmember extraction algorithm," IEEE Trans. Geosci. Remote Sens., vol. 44, no. 11, pp. 3397-3407, Nov. 2006.

[30] S.-S. Cai, Q. Du, and R. Moorhead, "Hyperspectral imagery visualization using double layers," IEEE Trans. Geosci. Remote Sens., vol. 45, no. 10, pp. 3028-3036, Oct. 2007.

[31] N. P. Jacobson, M. R. Gupta, and J. B. Code, "Linear fusion of image sets for display," IEEE Trans. Geosci. Remote Sens., vol. 45, no. 10, pp. $3277-$ 3288 , Oct. 2007.

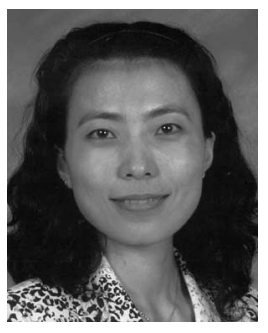

Qian Du (S'98-M'00-SM'05) received the Ph.D degree in electrical engineering from the University of Maryland, Baltimore County, in 2000.

She was with the Department of Electrical Engineering and Computer Science, Texas A\&M University-Kingsville, from 2000 to 2004. She joined the Department of Electrical and Computer Engineering at Mississippi State University, Mississippi State, in 2004, where she is currently an Associate Professor. Her research interests include remote sensing image analysis, pattern classification, data compression, and neural networks.

Dr. Du is a member of the Society of Photographic Instrumentation Engineers, the American Society of Photogrammetry and Remote Sensing, and the American Society for Engineering Education.

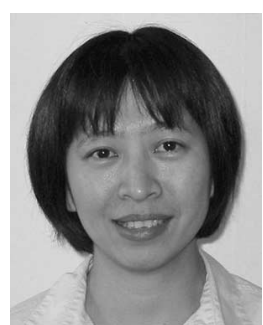

Nareenart Raksuntorn (S'06) received the B.E. degree in electronics from King Mongkut's Institute of Technology, Ladkrabang, Thailand, in 1994 and the M.S. degree in electrical engineering from the University of Colorado, Denver in 2002. She is currently pursuing the Ph.D. degree at Mississippi State University, Mississippi State. She is expected to graduate in December 2008.

Her research interests include remote sensing, image processing, and pattern recognition.

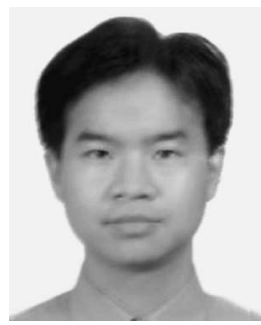

Shangshu Cai (S'06) received the B.S. and M.S. degrees in electrical engineering (Special Class for the Gifted Young) from the University of Science and Technology of China, Hefei, in 2000 and 2003, respectively. He is currently pursuing the Ph.D. degree at the Department of Electrical and Computer Engineering, Mississippi State University. He is expected to graduate in August 2008.

His research interests include scientific visualization, high-dimensional data visualization, hyperspectral image display, and image processing.

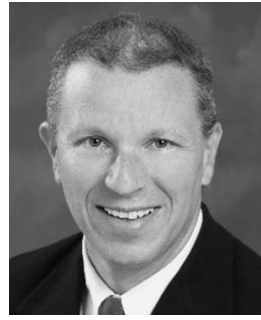

Robert J. Moorhead, II (S'81-M'85-SM'92) received the B.S.E.E. degree from Geneva College, Beaver Falls, PA, in 1980 and the Ph.D. degree in electrical and computer engineering from North Carolina State University in 1985.

$\mathrm{He}$ is currently a Professor in the Department of Electrical and Computer Engineering at Mississippi State University, Mississippi State, and Deputy Director of the GeoResources Institute, Mississippi State. He was previously a Research Staff Member at the IBM T.J. Watson Research Center. His current research interests include computationally demanding visualization and analysis issues. He has previously conducted research in computer communications and image/video coding and has published more than 100 papers. 